\title{
A Monotonicity Condition for Strong Convergence of the Mann Iterative Sequence for Demicontractive Maps in Hilbert Spaces
}

\author{
Akuchu Besheng George ${ }^{1}$, Celestin Akwumbuom Nse ${ }^{2}$ \\ ${ }^{1}$ Department of Mathematics, University of Nigeria, Nsukka, Nigeria \\ ${ }^{2}$ Department of Mathematics, Federal University of Technology, Owerri, Nigeria \\ Email: george.akuchu@unn.edu.ng, drcelestinse@yahoo.com
}

Received 18 May 2014; revised 22 June 2014; accepted 6 July 2014

Copyright (C 2014 by authors and Scientific Research Publishing Inc.

This work is licensed under the Creative Commons Attribution International License (CC BY).

http://creativecommons.org/licenses/by/4.0/

\section{(c) (i) Open Access}

\section{Abstract}

Let $H$ be a real Hilbert space and $C$ be a nonempty closed convex subset of $H$. Let $T: C \rightarrow C$ be a demicontractive map satisfying $\langle T x, x\rangle \geq\|x\|^{2}$ for all $x \in D(T)$. Then the Mann iterative sequence given by $x_{n+1}=\left(1-\alpha_{n}\right) x_{n}+\alpha_{n} T x_{n}$, where $\alpha_{n} \in(0,1) \forall n \geq 0$, converges strongly to an element of $F(T):=\{x \in C: T x=x\}$. This strong convergence is obtained without the compactnesstype assumptions on $C$, which many previous results (see e.g. [1]) employed.

\section{Keywords}

Demicontractive Maps, Mann Iterative Sequence, Strong Convergence, Monotonicity, Hilbert Spaces

\section{Introduction}

Let $H$ be a real Hilbert space. A mapping $T: H \rightarrow H$ is said to be demicontractive if there exists a constant $k>0$ such that

$$
\|T x-p\|^{2} \leq\|x-p\|^{2}+k\|x-T x\|^{2}
$$

for all $(x, p) \in H \times F(T)$, where $F(T):=\{x \in H: T x=x\} \neq \varnothing$. More often than not, $k$ is assumed to be in 
the interval $(0,1)$. However, this is a restriction of convenience. If $k=1$, then $T$ is called a hemicontractive map.

On the otherhand, $T$ is said to satisfy condition (A) if there exists $\lambda>0$ such that

$$
\langle x-T x, x-p\rangle \geq \lambda\|x-T x\|^{2},
$$

for all $(x, p) \in H \times F(T)$. Inequality (1.2) is equivalent to

$$
\langle T x-p, x-p\rangle \leq\|x-p\|^{2}-\lambda\|x-T x\|^{2} .
$$

The above classes of maps were studied independently by Hicks and Kubicek [2] and Maruster [3]. It is however shown in [4] that the two classes of maps coincide if $k \in(0,1)$ and $\lambda \in\left(0, \frac{1}{2}\right)$.

The class of demicontractive maps includes the class of quasi-nonexpansive and the class of strictly pseudocontractive maps. Any strictly pseudocontractive mapping with a nonempty fixed point set is demicontractive.

If $C$ is a closed convex subset of any Banach space $E$ and $T: C \rightarrow C$ is any map, then the Mann iteration sequence [5] is given by $x_{n+1}=\left(1-\alpha_{n}\right) x_{n}+\alpha_{n} T x_{n}$, where $\alpha_{n} \in(0,1) \forall n \geq 0$, satisfying certain conditions. Several authors (see e.g. [1]-[3] [5] [6]) have studied the convergence of the Mann iteration sequence to fixed points of certain mappings in certain Banach spaces. However, the Mann iteration sequence is very suitable for the study of convergence to fixed points of demicontractive mappings. It is well known (see e.g. [4]) that demicontractivity alone is not sufficient for the convergence of the Mann iteration sequence. Some additional smoothness properties of $T$ such as continuity and demiclosedness are necessary.

A map $T$ is said to be demiclosed at a point $x_{0}$ if whenever $\left\{x_{n}\right\}$ is a sequence in the domain of $T$ such that $\left\{x_{n}\right\}$ converges weakly to $x_{0} \in D(T)$ and $\left\{T x_{n}\right\}$ converges strongly to $y_{0}$, then $T x_{0}=y_{0}$.

In [7], Maruster studied the convergence of the Mann iteration sequence for demicontractive maps, in finite dimensional spaces, with an application to the study of the so-called relaxation algorithm for the solution of a particular convex feasibility problem. More precisely, he proved the following:

Theorem 1 [7]: Let $T: \mathfrak{R}^{m} \rightarrow \Re^{m}$ be a nonlinear mapping, where $\mathfrak{R}^{m}$ is the $m$-Euclidean space. Suppose the following are satisfied:

1) $I-T$ is demiclosed at 0 .

2) $T$ is demicontractive with constant $k$, or equivalently $T$ satisfies condition $A$ with $\lambda=\frac{1-k}{2}$.

Then the Mann iteration sequence converges to a point of $F(T)$ for any starting $x_{0}$.

Maruster [4] noted that in infinite dimensional spaces, demicontractivity and demiclosedness of $T$ are not sufficient for strong convergence. However, the two conditions ensure weak convergence. More precisely, he proved the following:

Theorem 2 [3]: Let $T: C \rightarrow C$ be a nonlinear mapping with $F(T) \neq \varnothing$, where $C$ is a closed convex subset of a real Hilbert space $H$. Suppose the following conditions are satisfied:

1) $I-T$ is demiclosed at 0 .

2) $T$ is demicontractive with constant $k$, or equivalently $T$ satisfies condition $A$ with $\lambda=\frac{1-k}{2}$.

3) $0<a \leq \alpha_{n} \leq b<2 \lambda=1-k$.

Then the Mann iteration sequence converges weakly to a fixed point of $F(T)$, for any starting $x_{0}$.

\section{Strong Convergence}

As noted above, demicontractivity and demiclosedness of $T$ are not sufficient for strong convergence of the Mann iteration sequence in infinite dimensional spaces. Some additional conditions on $T$, or some modifications of the Mann iteration sequence are required for strong convergence to fixed points of demicontractive maps. Such additional conditions or modifications have been studied by several authors (see e.g. [1] [2] [6] [8] [9]).

There is however an interesting connection between the strong convergence of the Mann iteration sequence to a fixed point of a demicontractive map, $T$, and the existence of a non-zero solution of a certain variational inequality. This connection was observed by Maruster [3], and has been studied by several authors. More precisely, 
Maruster proved the following theorem:

Theorem 3 [3]: Suppose $T$ satisfies the conditions of Theorem 2. If in addition there exists $0 \neq h \in H$ such that

$$
\langle x-T x, h\rangle \leq 0
$$

for all $x \in D(T)$, then starting from a suitable $x_{0}$, the Mann iteration sequence converges strongly to an element of $F(T)$.

The conditions of/and the variational inequality in Theorem 3 have been used and generalized by several authors (see e.g. [8] [9]). The existence of a non-zero solution to the variational inequality is sometimes gotten under very stringent conditions. In [4] remark 4, Maruster and Maruster made the following observation "It would therefore be interesting to study more closely the existence of a non-zero solution of the variational inequality".

The purpose of this paper is to provide a monotonicity condition under which the Mann iteration sequence converges strongly to a fixed point of a demicontractive map. The convergence does not need to pass through the variational inequality (1.4). The condition is embodied in the following theorem:

Before we state and prove our theorem, we give the following definition which will be useful in the sequel.

Definition 1: Let $H$ be a real Hilbert space with inner product $\langle.,$.$\rangle and norm \|$.$\| and let C$ be a nonempty closed convex subset of $H$. The orthogonal projection $P_{C} x$ of $H$ onto $C$ is defined by $P_{C} x=\arg \min _{y \in C}\|x-y\|$, and has the following properties:

1) $\left\langle x-P_{C} x, z-P_{C} x\right\rangle \leq 0$, for all $z \in C$.

2) $\left\|P_{C} x-P_{C} y\right\|^{2} \leq\left\langle P_{C} x-P_{C} y, x-y\right\rangle$, for all $x, y \in H$.

Theorem 4: Suppose $T$ satisfies:

1) The conditions of Theorem 2.

2) $\langle T x, x\rangle \geq\|x\|^{2}$ for all $x \in D(T)$. Then starting from a suitable $x_{0}$, the Mann iteration sequence converges strongly to an element of $F(T)$.

Proof. Choose $x_{0} \in C$ such that $\left\langle x_{0}-p, p\right\rangle \geq 0$. This implies there exists $\epsilon_{0}>0$ such that $\left\langle x_{0}-p, p\right\rangle \geq \epsilon_{0}\left\|x_{0}-p\right\|^{2}$. Suppose $\left\langle x_{n}-p, p\right\rangle \geq \epsilon_{0}\left\|x_{n}-p\right\|^{2}$. Then using (1.3) and condition (ii) of Theorem 4, we have

$$
\begin{aligned}
\left\langle x_{n+1}-p, p\right\rangle & =\left\langle\left(1-\alpha_{n}\right)\left[x_{n}-p\right]+\alpha_{n}\left[T x_{n}-p\right], p\right\rangle \\
& =\left(1-\alpha_{n}\right)\left\langle x_{n}-p, p\right\rangle+\alpha_{n}\left\langle T x_{n}-p, p\right\rangle \\
& =\left(1-\alpha_{n}\right)\left\langle x_{n}-p, p\right\rangle+\alpha_{n}\left\langle T x_{n}-x_{n}, p\right\rangle+\alpha_{n}\left\langle x_{n}-p, p\right\rangle \\
& =\left\langle x_{n}-p, p\right\rangle+\alpha_{n}\left\langle T x_{n}-x_{n}, p\right\rangle \\
& =\left\langle x_{n}-p, p\right\rangle+\alpha_{n}\left[\left\langle T x_{n}-p+p-x_{n},-x_{n}+p\right\rangle+\left\langle T x_{n}-x_{n}, x_{n}\right\rangle\right] \\
& =\left\langle x_{n}-p, p\right\rangle-\alpha_{n}\left\langle T x_{n}-p, x_{n}-p\right\rangle+\alpha_{n}\left\langle x_{n}-p, x_{n}-p\right\rangle+\alpha_{n}\left\langle T x_{n}-x_{n}, x_{n}\right\rangle \\
& \geq\left\langle x_{n}-p, p\right\rangle-\alpha_{n}\left[\left\|x_{n}-p\right\|^{2}-\lambda\left\|x_{n}-T x_{n}\right\|^{2}\right]+\alpha_{n}\left\|x_{n}-p\right\|^{2}+\alpha_{n}\left\langle T x_{n}-x_{n}, x_{n}\right\rangle \\
& =\left\langle x_{n}-p, p\right\rangle+\lambda \alpha_{n}\left\|x_{n}-T x_{n}\right\|^{2}+\alpha_{n}\left\langle T x_{n}-x_{n}, x_{n}\right\rangle \\
& \geq\left\langle x_{n}-p, p\right\rangle \geq \epsilon_{0}\left\|x_{n}-p\right\|^{2} \geq \epsilon_{0}\left\|x_{n+1}-p\right\|^{2}
\end{aligned}
$$

Since $x_{n} \rightarrow p$ from Theorem 2, then $x_{n} \rightarrow p$.

Example: Let $H=R$ (reals) and $C=[0,2]$ be a nonempty closed convex subset of $H$. Define $T: C \rightarrow C$ by

$$
T x= \begin{cases}2, & \text { if } x<\frac{1}{3} \\ x-\frac{5}{2}(x-1), & \text { if } \frac{1}{3} \leq x<1 \\ x, & \text { if } x \geq 1\end{cases}
$$


Then it is easily verifiable that $T$ is demicontractive and satisfies condition (ii) of our theorem for

$$
0<\lambda \leq \frac{2}{5}
$$

Remark 1: In [4] Maruster and Maruster noted that if $T$ satisfies the positivity type condition $\langle T x, x\rangle \geq\|x\|^{2}$, then it is sufficient to find a non-zero solution of the variational inequality (1.4). This motivates the condition in our theorem. As a matter of fact, our theorem is a necessity result.

Remark 2: We note that one of the ways of choosing $x_{0}$ is as follows: For any $\beta>1$, choose $x_{0}=P_{C}(\beta p)$, where $p \in F(T)$ and $P: H \rightarrow C$ is the metric projection from $H$ into $C$. This follows since it is well known (see Definition 1 ) that $P$ is firmly nonexpansive (i.e. satisfies condition (ii) of Definition 1), so that

$$
\begin{aligned}
\left\|x_{0}-p\right\|^{2} & =\left\|P_{C}(\beta p)-P(p)\right\|^{2} \leq\left\langle P_{C}(\beta p)-P_{C}(p), \beta p-p\right\rangle \\
& =\left\langle x_{0}-p,(\beta-1) p\right\rangle=(\beta-1)\left\langle x_{0}-p, p\right\rangle
\end{aligned}
$$

This implies $\left\langle x_{0}-p, p\right\rangle \geq \varepsilon_{0}\left\|x_{0}-p\right\|^{2}$, where $\varepsilon_{0}=\frac{1}{\beta-1}$.

\section{References}

[1] Rafiq, A. (2007) On the Mann Iteration in Hilbert Spaces. Nonlinear Analysis, 66, 2230-2236. http://dx.doi.org/10.1016/j.na.2006.03.012

[2] Hicks, H.L. and Kubicek, J.D. (1977) On the Mann Iteration in Hilbert Spaces. Journal of Mathematical Analysis and Applications, 59, 498-505. http://dx.doi.org/10.1016/0022-247X(77)90076-2

[3] Maruster, St. (1973) Sur le Calcul des Zeros d'un Operateur Discontinu par Iteration. Canadian Mathematical Bulletin, 16, 541-544. http://dx.doi.org/10.4153/CMB-1973-088-7

[4] Maruster, L. and Maruster, S. (2011) Strong Convergence of the Mann Iteration for $\alpha$-Demicontractive Mappings. Mathematical and Computer Modelling, 54, 2486-2492. http://dx.doi.org/10.1016/j.mcm.2011.06.006

[5] Mann, W. (1953) Mean Value Methods in Iteration. Proceedings of the American Mathematical Society, 4, 506-510. http://dx.doi.org/10.1090/S0002-9939-1953-0054846-3

[6] Maruster, St. (1977) The Solution by Iteration of Nonlinear Equations in Hilbert Spaces. Proceedings of the American Mathematical Society, 63, 767-773. http://dx.doi.org/10.1090/S0002-9939-1977-0636944-2

[7] Chidume, C.E. and Maruster, St. (2010) Iterative Methods for the Computation of Fixed Points of Demicontractive Mappings. Journal of Computational and Applied Mathematics, 234, 861-882. http://dx.doi.org/10.1016/j.cam.2010.01.050

[8] Osilike, M.O. (2000) Strong and Weak Convergence of the Ishikawa Iteration Method for a Class of Nonlinear Equations. Bulletin of the Korean Mathematical Society, 37, 153-169.

[9] Chidume, C.E. (1984) The Solution by Iteration of Nonlinear Equations in Certain Banach Spaces. Journal of the Nigerian Mathematical Society, 3, 57-62. 
Scientific Research Publishing (SCIRP) is one of the largest Open Access journal publishers. It is currently publishing more than 200 open access, online, peer-reviewed journals covering a wide range of academic disciplines. SCIRP serves the worldwide academic communities and contributes to the progress and application of science with its publication.

Other selected journals from SCIRP are listed as below. Submit your manuscript to us via either submit@scirp.org or Online Submission Portal.
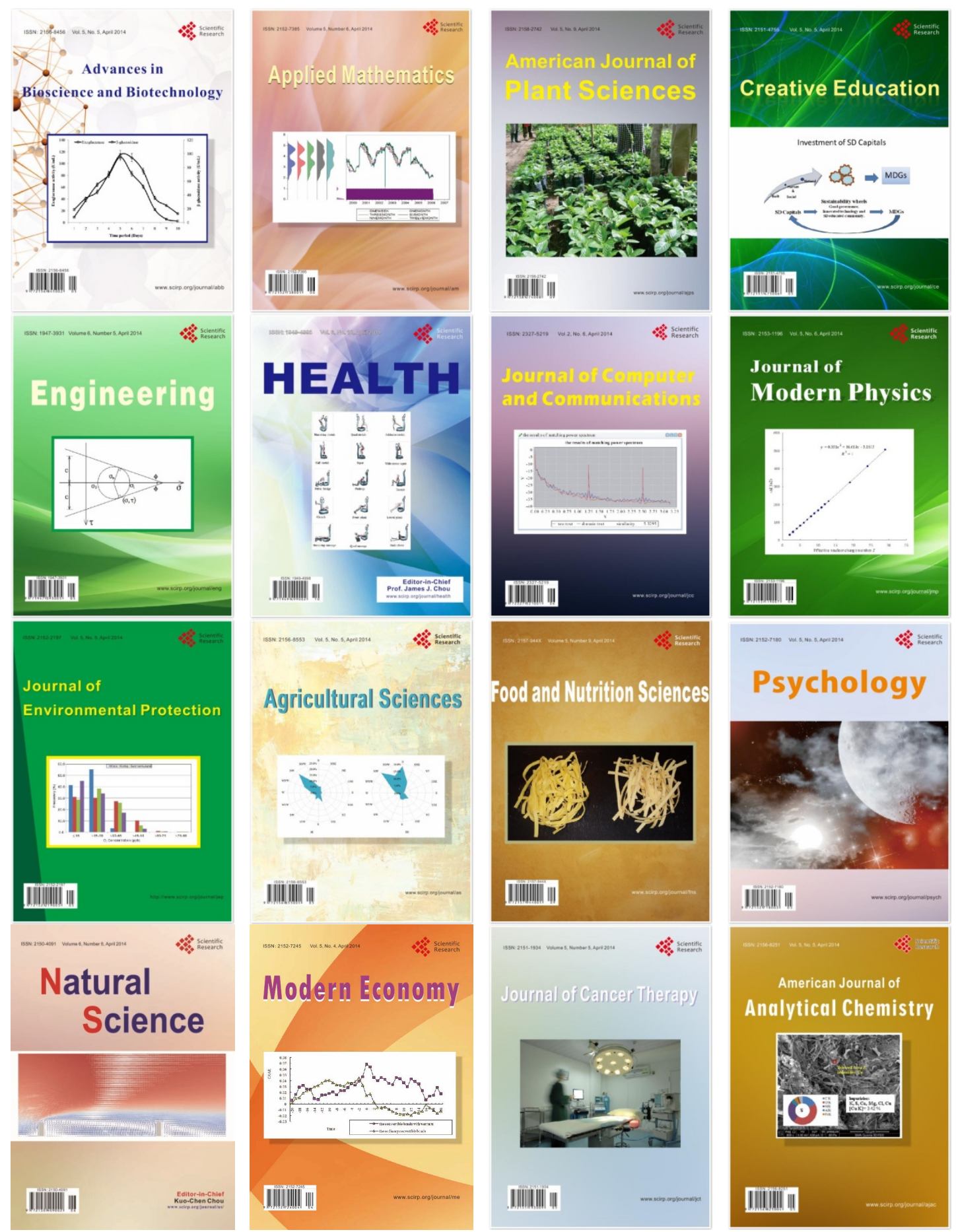